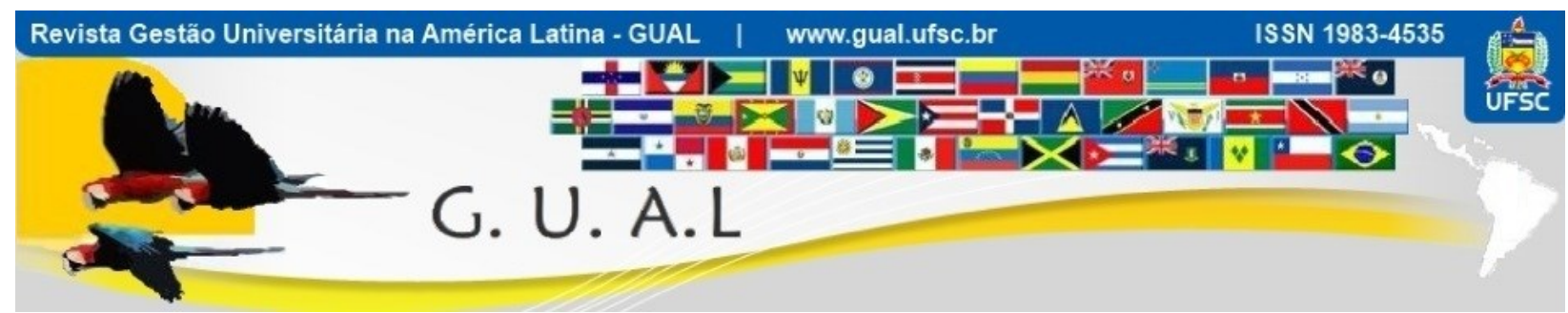

DOI: http://dx.doi.org/10.5007/1983-4535.2019v12n3p151

\title{
AVALIAÇÃO ESTRATÉGICA DA PRODUTIVIDADE ACADËMICA DE DOCENTES DE CURSOS DE GRADUAÇÃO
}

\section{STRATEGIC ASSESSMENT OF ACADEMIC PRODUCTIVITY OF BRAZILIAN PROFESSORS IN UNDERGRADUATE COURSES}

\author{
Marcus Vinicius Cesso, Mestre \\ https://orcid.org/0000-0001-9204-7049 \\ marcus.cesso@gmail.com \\ Universidade Nove de Julho | Programa de Pós-Graduação em Administração \\ São Paulo | São Paulo | Brasil \\ Renato Ribeiro Nogueira Ferraz, Doutor \\ https://orcid.org/0000-0002-8931-895X \\ renatobio@hotmail.com \\ Universidade Mogi das Cruzes | Programa de Pós-Graduação em Políticas Públicas \\ Mogi das Cruzes | São Paulo | Brasil
}

Recebido em 11/setembro/2018

Aprovado em 08/abril/2019

Publicado em 02/setembro/2019

Sistema de Avaliação: Double Blind Review

Revista GUAL, Florianópolis, v. 12, n. 3, p. 151-172, setembro-dezembro 2019 www.gual.ufsc.br 


\title{
RESUMO
}

As Instituições de Ensino Superior (IES) brasileiras têm seus cursos de graduação avaliados pelo Instituto Nacional de Estudos e Pesquisas Educacionais Anísio Teixeira (INEP), periodicamente. Entre os critérios definidos, destaca-se a dimensão Corpo Docente e Tutorial, que corresponde a $30 \%$ da nota total dos cursos de graduação. Assim, o estudo utiliza a ferramenta computacional Scriptemec para a avaliação contínua e automatizada da produção acadêmica dos cursos de graduação em Enfermagem e Medicina, de uma IES particular. Com o uso de uma metodologia mista de pesquisa, foram avaliados os critérios de produtividade acadêmica, como produção bibliográfica, produção técnica, e popularização dos resultados das pesquisas realizadas pelos docentes da instituição, de forma quantitativa e qualitativa, permitindo uma análise longitudinal dos dados em um longo período de tempo. Como contribuição do presente estudo, reforça-se a possibilidade do estudo do desempenho acadêmico em pesquisa de docentes de cursos de graduação com o apoio de uma ferramenta estratégica de livre acesso e que permite uma otimização na análise de indicadores de produtividade intelectual, além da construção de um dashboard automatizado com os dados de produtividade acadêmica dos docentes, possibilitando o acompanhamento contínuo destes indicadores para prestação de contas ao INEP e avaliação interna da instituição.

Palavras-chave: Scriptemec. Produtividade Acadêmica. Avaliação do Ensino Superior. Gestão Universitária.

\begin{abstract}
The Brazilian Higher Education Institutions (HEI) have their undergraduate courses evaluated periodically by the National Institute of Studies and Educational Research Anísio Teixeira (INEP). Among the criteria defined by INEP, highlight the Faculty and Tutorial dimension, which corresponds to $30 \%$ of the total grade of HEI undergraduate courses. Thus, the present study aims to use the computational tool Scriptemec for the continuous and automated evaluation of the academic production of undergraduate courses in Nursing and Medicine, from a private HEI. Applying a mixed methodology, the academic productivity criteria, such as bibliographic production, technical production, and popularization of the research results of the institution's professors were evaluated in a quantitative and qualitative approach, allowing a longitudinal analysis of the data in a long period of time. As a contribution of the present study is reinforced the possibility of studying the academic performance in undergraduate teaching research with the support of a strategic tool of free access, allowing an optimization in the analysis of indicators of intellectual productivity, besides the construction of an automated dashboard with the academic productivity data of the professors, enabling the continuous monitoring of these indicators to render accounts to the INEP and internal evaluation of the institution.
\end{abstract}

Keywords: Scriptemec. Academic Productivity. Higher Education Assessment. University Management. 


\section{INTRODUÇÃO}

As instituições de ensino superior brasileiras - IES - estão inseridas em um ambiente de alta concorrência, especialmente devido às constantes mudanças relacionadas aos seus clientes internos e externos, além das políticas educacionais estabelecidas pelos órgãos governamentais (MACCARI et al., 2014). Devido a este cenário de constante pressão sobre as organizações educacionais, as direções das IES buscam uma gestão estratégica com base em seus recursos, a fim de obter uma maior eficiência organizacional e melhora no desempenho deste modelo de organização (MARTINS et al., 2013). Entretanto, os modelos de gestão ainda se mantêm muito próximos dos praticados em outros tipos de organizações (BAETA; BRITO; SOUZA, 2014), o que não se torna efetivo no segmento de ensino superior, que apresenta características institucionais e políticas exclusivas (MEYER; PASCUCCI; ANDRIGUETTO, 2017; SOARES; ROMAN; ERDMANN, 2016). Tais políticas são constantemente avaliadas por órgãos governamentais, como o Instituto Nacional de Estudos e Pesquisas Educacionais Anísio Teixeira - INEP (POLIDORI et al., 2011), que está vinculado ao Ministério da Educação - MEC.

O INEP promove, periodicamente, avaliações dos cursos de graduação das IES brasileiras, a fim de verificar a qualidade do Projeto Didático-Pedagógico, Infraestrutura, e Formação do Corpo Docente e Tutorial (POLIDORI et al., 2011; SOBRINHO, 2010). Essa última dimensão, Formação do Corpo Docente e Tutorial, está ligada à principal missão das IES, que é a disseminação do conhecimento por meio do ensino e da pesquisa. Ademais, esse quesito representa $30 \%$ da nota final da avaliação dos cursos de graduação, sendo essencial para a mensuração da qualidade da IES (HOFFMANN et al., 2014). Assim, Marchelli (2007) reforça que os indicadores relacionados a produtividade acadêmica do corpo docente das IES merecem mais atenção no momento da avaliação dos cursos de graduação. Corroborando com esse apontamento, Polidori e Carvalho (2016) justificam que estas organizações buscam a acreditação de qualidade de seus cursos de graduação à partir do reforço do ensino e pesquisa promovidos por elas, além do preenchimento correto dos instrumentos avaliativos (SOBRINHO, 2010), que para cursos de graduação deve ser feito por meio da Plataforma $e$ MEC.

Para aperfeiçoar o preenchimento desses instrumentos avaliativos do ensino superior brasileiro, automatizando o processo, diversos autores recomendam a utilização de ferramentas computacionais, que auxiliam os gestores acadêmicos na inserção das 
informações nestas plataformas de avaliação (ALVES; SANTOS; SCHIMIT, 2016; FERRAZ et al., 2017; MENA-CHALCO; JUNIOR, 2009; SILVA; FERRAZ, 2017), destacando o estudo de Silva (2017), que apresenta e justifica a utilização da ferramenta Scriptemec, dedicada à avaliação da produtividade acadêmica de pesquisadores ligados a cursos de graduação, sendo um instrumento de gestão de desempenho docente, e discente, por meio de indicadores, que pode ser utilizado pelos gestores acadêmicos das IES.

Com base no problema até então apresentado, este trabalho pretende responder a seguinte questão de pesquisa, que norteará o seu desenvolvimento: "Como o Scriptemec auxilia na avaliação do desempenho em produtividade acadêmica de docentes nos cursos de graduação das IES brasileiras?"

Neste contexto, o objetivo principal deste artigo é apresentar um modelo de avaliação do desempenho de docentes dos cursos de graduação, atendendo aos indicadores propostos pelo INEP, e relacionados ao pilar Corpo Docente e Tutorial. Com isso, pretende-se demonstrar que com a utilização do Scriptemec é possível acompanhar a produtividade acadêmica dos docentes ao longo de um período desejado, avaliar os níveis de transparência/popularização das pesquisas, e realizar a análise da responsabilidade social dos estudos promovidos pelos pesquisadores ligados aos cursos de graduação de IES brasileiras.

Além desta Introdução, segue-se a seção de Referencial Teórico, em que serão dispostos os principais pilares teóricos que buscaram sustentar este trabalho. Na sequência, será apresentada a seção de Métodos da Pesquisa, em que se demonstra os procedimentos de coleta e análise dos dados do presente estudo, além da caracterização da IES que será utilizada como objeto desta experimentação. Na seção de Resultados, apresentam-se os dados gerados pelo Scriptemec relacionados a produtividade acadêmica dos docentes analisados. Já na seção de Discussão, serão debatidos os resultados obtidos, sob a luz da literatura. Por fim, na seção de Considerações Finais, argumenta-se sobre a utilização do Scriptemec como ferramenta estratégica para a avaliação do desempenho em produtividade acadêmica de docentes de cursos de graduação de IES brasileiras e a criação de um dashboard para a avaliação contínua e automatizada dos indicadores apresentados neste estudo. 


\section{REFERENCIAL TEÓRICO}

\subsection{SISTEMA DE AVALIAÇÃO DO ENSINO SUPERIOR BRASILEIRO E DOS CURSOS DE GRADUAÇÃO}

A avaliação do ensino superior brasileiro já é um tema debatido desde a década de 1950, quando foi criada a CAPES, que tinha como objetivo principal aprimorar a qualidade do serviço de ensino prestado pelas IES, focando-se nos cursos de Pós-Graduação Stricto sensu (CAPES, 2016). Na década de 1970, esta avaliação do ensino superior brasileiro se intensificou, devido ao crescimento no número de cursos de Mestrado e Doutorado no Brasil (MACCARI et al., 2014).

No entanto, somente a partir de 1980, trinta anos após o sistema brasileiro de ensino superior começar a receber a atenção de órgãos focados na mensuração da qualidade dos serviços prestados, é que os cursos de graduação começaram a ser avaliados (SOBRINHO, 2010). As avaliações foram iniciadas pelo MEC, com base em uma série de ferramentas, como por exemplo, o Programa de Avaliação da Reforma Universitária - PARU, a constituição da "Comissão de Notáveis" e do Grupo Executivo da Reforma da Educação Superior - GERES (POLIDORI et al., 2011).

Após estas primeiras iniciativas para a avaliação dos cursos de graduação no Brasil, exclusivamente por meio de ações governamentais dirigidas pelo MEC, as IES começaram a se organizar para definir instrumentos que promovessem sua autoavaliação que, conforme Polidori e Carvalho (2016), foi uma forma de acreditação e autoconhecimento desenvolvido por este tipo de organização. A partir da autoavaliação, as IES passaram a organizar seus dados para que pudessem obter seus indicadores e, assim, estabelecer um modelo de apresentação de resultados de qualidade, que atendessem as recomendações dos órgãos governamentais responsáveis pela mensuração da qualidade do Ensino Superior brasileiro (HOFFMANN et al., 2014).

Após as iniciativas de autoavaliação promovidas pelas IES se estabelecerem como forma adequada para mensuração da qualidade do ensino superior brasileiro, criou-se o Programa de Avaliação Institucional das Universidades Brasileiras - PAIUB, regulado pelo MEC. Porém, mesmo com o fortalecimento deste novo modelo, o PAIUB, ainda não apresentava critérios de mensuração da qualidade dos serviços de ensino e pesquisa parametrizados entre todas as IES brasileiras, permitindo que cada organização estabelecesse indicadores diferentes, o que não possibilitava um alinhamento sobre a qualidade dos cursos 
de graduação no Brasil (SOBRINHO, 2010). Por isso, devido ao PAIUB não ser uma ferramenta completa de avaliação, entre 1996 e 1997, foi criado o Exame Nacional de Cursos - ENC, conhecido como "Provão". Este novo modelo de avaliação tinha como objetivo principal regulamentar o crescimento institucional e mensurar o desempenho de todas as IES brasileiras (BARREYRO, 2008; POLIDORI et al., 2011). Somente após o "Provão", o MEC conseguiu estabelecer os critérios para autorização e reconhecimento dos cursos de graduação no Brasil. Contudo, com o objetivo de tornar ainda mais completa a avaliação destes cursos, o MEC desenvolveu mais duas ferramentas que complementariam o "Provão", que foram a Avaliação das Condições de Ensino e a Avaliação Institucional (MEC, 2017; SOBRINHO, 2010).

No entanto, mesmo com um sistema composto de três ferramentas, o MEC considerou que nem todas as IES conseguiriam estabelecer parâmetros de qualidade que permitissem uma avaliação completa e única, entre todas as instituições. Por isso, em 2004, foi criado o Sistema Nacional de Avaliação do Ensino Superior - SINAES, com a missão de ser um sistema unificado com parâmetros bem determinados para promover uma avaliação completa em todas as IES, cumprindo as definições estabelecidas pelos órgãos governamentais que mensuram a qualidade do ensino superior no Brasil (LACERDA; FERRI; DUARTE, 2016; POLIDORI; CARVALHO, 2016; SOBRINHO, 2010). Portanto, o SINAES, que está sob a coordenação do INEP, consegue atender os parâmetros de qualidade definidos pela Comissão Nacional de Avaliação da Educação Superior - CONAES (CANAN; ELOY, 2016), estabelecendo três eixos para mensuração da qualidade do ensino: a avaliação institucional das IES, do desempenho dos discentes, e dos cursos de graduação de uma IES. Estas dimensões podem ser vistas, de forma resumida, na Figura 1.

Figura 1 Eixos para mensuração da qualidade do ensino superior brasileiro estabelecido pelo SINAES

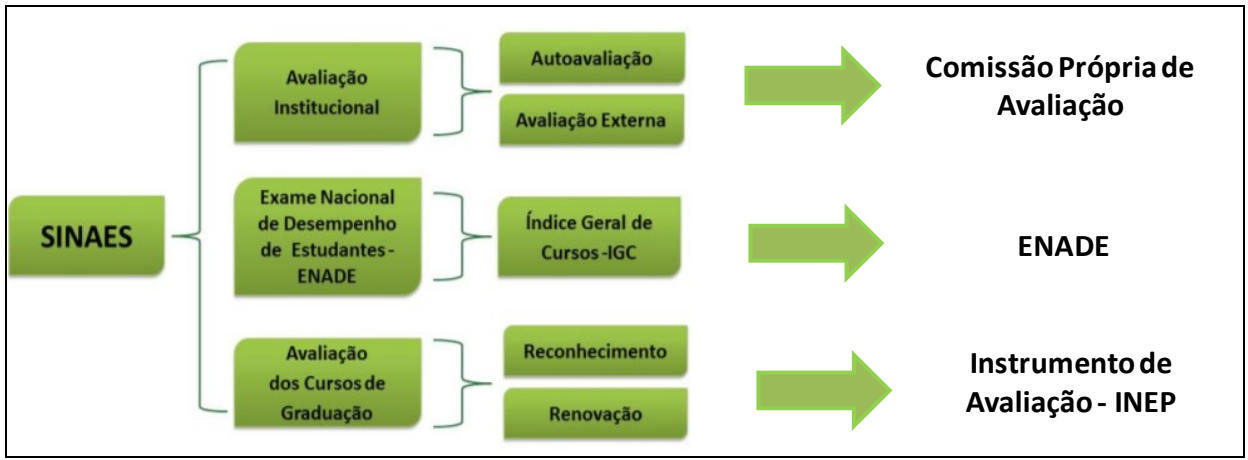

Fonte: adaptado de MEC (2017). 
O primeiro eixo, que é a Avaliação Institucional das IES, atende aos critérios definidos pela Lei $n^{o} 10.861 / 04$ e pela portaria $n^{0} 2051$ de 09/07/2004 (MEC, 2017), que tornou obrigatória a todas as IES a criação de suas Comissões Próprias de Avaliação - CPA. A comissão é um instrumento interno de avaliação institucional que, embora seja oriundo de uma regulamentação governamental, possui um papel essencial no processo de mensuração da qualidade de ensino e pesquisa das IES (SANTO; TRAVASSOS; CARIBÉ, 2016). Além disso, por ser uma ferramenta utilizada internamente, as IES conseguem planejar ações, conforme sua avaliação dos indicadores levantados pela CPA, para melhorar o desempenho discente e docente (POLIDORI et al., 2011), sua infraestrutura e responsabilidade social (BERNARDES; ROTHEN, 2016), e sua sustentabilidade financeira (GOMES et al., 2015).

O segundo eixo, a avaliação do desempenho discente, se utiliza do Exame Nacional de Desempenho de Estudantes - ENADE, para avaliar a formação técnico-acadêmica dos alunos egressos de uma IES (MEC, 2017). Este exame se trata de uma avaliação que consegue atender as necessidades do corpo discente das IES, relacionando a teoria acadêmica com a prática profissional (POLIDORI et al., 2011), a autenticação do conhecimento obtido durante todo o curso de graduação (CASTRO et al., 2016), o alinhamento dos conhecimentos técnicocientíficos do discente em relação a seus pares do Brasil (BRITO, 2008; SILVA; FERRAZ, 2018; SOBRINHO, 2010), e o reconhecimento da qualidade dos cursos de graduação da IES pelos egressos (CANAN; ELOY, 2016; HOFFMANN et al., 2014; POLIDORI; CARVALHO, 2016).

Assim, o ENADE possui um papel estratégico nas IES, possibilitando que estas instituições promovam ações que melhorem a qualidade dos serviços de ensino e pesquisa prestados, incrementem os resultados das avaliações institucionais, e aprimorem a precisão dos dados que são apresentados ao INEP, periodicamente (CESSO; FERRAZ, 2017).

Mesmo com uma avaliação interna, feita pela CPA, e uma avaliação focada no desempenho dos alunos, mensurada pelo ENADE, as IES passam por um processo externo de avaliação, promovido por visitas in loco de especialistas designados pelo MEC (SOBRINHO, 2010). Estas comissões externas têm como objetivo avaliar as condições de ensino prestadas pelas IES, para auxiliar na autorização ou reconhecimento dos cursos de graduação (HOFFMANN et al., 2014).

Esta prática de avaliações externas para mensuração da qualidade dos cursos de graduação ocorre em diversos locais do mundo, como na Inglaterra, que possui um sistema de 
avaliação externa extremamente desenvolvida (CARDOSO; ROSA; STENSAKER, 2016), e na Índia, que possui a organização do ensino superior muito semelhante à brasileira, além de ser o segundo maior sistema de educação superior do mundo (MARTIN, 2016). Por isso, tanto em países desenvolvidos economicamente, assim como em nações com economias em desenvolvimento, a utilização de sistemas de avaliação externa dos cursos de graduação é uma forma de mensuração da qualidade de ensino prestado pelas IES (HOFFMANN et al., 2014; MARCHELLI, 2007).

Assim, no Brasil, o terceiro eixo praticado pelo SINAES - a avaliação externa dos cursos de graduação - é um instrumento válido e que sistematiza uma grande quantidade de informações, com base em indicadores que permitem a acreditação da qualidade dos serviços prestados pelas IES (POLIDORI; CARVALHO, 2016). Ademais, a utilização da comissão externa de avaliação permite uma transparência no processo avaliativo dos cursos de graduação, além de uma confirmação dos resultados obtidos por meio dos outros dois eixos de avaliação.

Dessa forma, a avaliação externa dos cursos de graduação promovida por comissões externas, selecionadas e qualificadas pelo MEC, tem uma excelência na mensuração da qualidade das IES semelhante aos principais sistemas do mundo, como ocorre na Inglaterra (CARDOSO; ROSA; STENSAKER, 2016) e na Índia (MARTIN, 2016). Ainda assim, diversos autores (BARREYRO, 2008; FERREIRA et al., 2016; LACERDA; FERRI; DUARTE, 2016; POLIDORI et al., 2011; SOBRINHO, 2010) discutem que o SINAES precisa ser revisto, tanto no âmbito político como em sua formatação e aplicação, para que apresente melhorias reais na mensuração da qualidade dos serviços prestados pelas IES. Ademais, Marchelli (2007) reforça que os pilares Titulação, Regime de Trabalho, e Publicações e Produções do Corpo Docente, presentes na dimensão Corpo Docente e Tutorial, são os principais indicadores que devem ser revistos no SINAES, devido a sua aderência ao objetivo principal das IES, que é disseminar o conhecimento por meio do ensino e pesquisa a toda comunidade (MEC, 2017).

Por isso, se entende que na avaliação feita pelo INEP, com o objetivo de autorizar ou reconhecer os cursos de graduação, a dimensão Corpo Docente e Tutorial deve ter maior representatividade, por apresentar maior aderência à missão principal das IES (MARTINS et al., 2013; MEYER; PASCUCCI; ANDRIGUETTO, 2017), permitindo sua acreditação junto à sociedade (POLIDORI; CARVALHO, 2016), e pelo fato de que nesta dimensão estão 
dispostos os principais recursos que permitem uma vantagem competitiva às IES (BARNEY, 1991), perante o mercado de alta concorrência ao qual estão inseridas (HOFFMANN et al., 2014), melhorando seu desempenho organizacional por meio da gestão de indicadores (GOMES et al., 2015).

\subsection{USO DE FERRAMENTAS COMPUTACIONAIS PARA GESTÃO DA PRODUTIVIDADE DOCENTE}

Com um processo isomórfico coercitivo (DIMAGGIO; POWELL, 2005) de avaliação dos cursos de graduação no Brasil, em que as IES são obrigadas a apresentar os resultados de indicadores, previamente estabelecidos por órgãos políticos que mensuram a qualidade dos serviços de ensino prestados por estas organizações (POLIDORI; CARVALHO, 2016), os gestores acadêmicos se utilizam do corpo técnico-administrativo para a coleta e organização dos dados a serem apresentados ao INEP. Neste contexto, para o gerenciamento e avaliação da produtividade acadêmica, as IES têm designado secretários-executivos (SILVA, 2017), que acessam a Plataforma Lattes, para coletar os dados dos pesquisadores, que compõe o corpo docente dos cursos de graduação.

A Plataforma Lattes é um banco de dados transparente, de acesso livre e público, para a consulta de informações de pesquisadores, grupos de pesquisa, IES, agências de pesquisa, e outras organizações que fomentem a ciência e tecnologia, permitindo o intercâmbio de conhecimento, e sendo uma fonte para gestão dos dados de pesquisa do Brasil, com mais de 3,5 milhões de currículos cadastrados (CNPQ, 2016). Ressalta-se que a Plataforma Lattes é uma base pública de inserção e recuperação de dados de pesquisa (ALVES; YANASSE; SOMA, 2012), em que diversas IES têm buscado integrar as informações obtidas por meio da plataforma com seus sistemas de gestão (CNPQ, 2016), gerando indicadores internos de produção intelectual (FERRAZ et al., 2017), que permitam o gerenciamento de suas políticas de ensino e pesquisa (RODRIGUES et al., 2017).

Assim, após esta coleta realizada por profissionais técnicos das IES, os dados são tabulados em planilhas e, posteriormente, organizados de forma manual, dentro do modelo estabelecido pelo INEP, para avaliação (POLIDORI et al., 2011). Todo este processo é suscetível a erros, já que é feito de forma manual, e pode acarretar prejuízos na avaliação das IES, gerando como consequências sanções promovidas pelo INEP, como por exemplo, a redução na nota dos cursos de graduação, que varia de 1 a 5 , conforme critérios estabelecidos 
no instrumento Avaliação de Cursos de Graduação presenciais e a distância, e auditados pelo MEC (POLIDORI; CARVALHO, 2016).

Com o objetivo de solucionar os possíveis erros manuais passíveis neste processo de coleta de dados da Plataforma Lattes, Silva e Ferraz (2017) apresentaram as quatro principais ferramentas computacionais que auxiliam os gestores acadêmicos das IES a gerenciarem a produtividade acadêmica do corpo docente de uma IES: o Lattes Extrator e o Lattes Miner (ALVES; YANASSE; SOMA, 2012), o Scriptlattes (MENA-CHALCO; JUNIOR, 2009) e o Scriptsucupira (FERRAZ et al., 2017).

Dentre as principais ferramentas que se propõem a automatizar o processo de extração, organização e apresentação de indicadores de produtividade em pesquisa, com base nos dados cadastrados na Plataforma Lattes, destacam-se o Scriptlattes e o Scriptsucupira, que são ferramentas computacionais de fonte aberta. A diferença entre as ferramentas se dá, principalmente, pela complexidade da análise que cada uma permite. O Scriptlattes possibilita que apenas uma lista de pesquisadores seja avaliada pontualmente, enquanto o Scriptsucupira executa vários Scriptlattes de forma concomitante, o que permite uma avaliação da produtividade acadêmica, de forma mais ampla e de um período maior, com base em várias listas de pesquisadores fornecidas à ferramenta computacional no início do processo de extração de dados (ALVES; SANTOS; SCHIMIT, 2016).

Diversos estudos já apresentaram a aplicação prática tanto do Scriptlattes quanto do Scriptsucupira em cursos de Pós-Graduação Stricto sensu (SILVA; FERRAZ, 2017), entretanto, ainda há poucos estudos sobre a avaliação de cursos de graduação utilizando ferramentas computacionais (SILVA, 2017), que pode ser explicada pelo fato de que o Scriptlattes e o Scriptsucupira foram desenvolvidas para atender, inicialmente, aos critérios estabelecidos pela CAPES, que avalia apenas os Programas de Mestrado e Doutorado no Brasil. Sendo assim, mesmo que estas ferramentas fossem utilizadas para avaliar um curso de graduação, os resultados obtidos ainda não atenderiam aos requisitos solicitados pelo INEP (MEC, 2017; POLIDORI et al., 2011).

Portanto, para que os gestores acadêmicos dos cursos de graduação possam cumprir os critérios estabelecidos pelo INEP, com base na dimensão Corpo Docente e Tutorial, que avalia a produção acadêmica de todos os professores das IES em nível de graduação, é necessária a utilização de uma ferramenta exclusiva para este modelo de curso, e que funcione de maneira semelhante à forma que o Scriptsucupira atua junto aos Programas de Pós- 
Graduação Stricto sensu. Dessa forma, Bavaresco (2017) apresentou uma ferramenta denominada Scriptemec para auxiliar o acompanhamento da produção intelectual de um grupo de docentes de cursos de graduação de uma IES, denominada Scriptemec.

O Scriptemec é uma ferramenta computacional open source, semelhante ao Scriptsucupira, desenvolvida para a plataforma Linux, que a partir da definição de uma lista prévia de professores de cursos de graduação, realiza a extração e organização dos dados acadêmicos e profissionais registrados por estes professores na Plataforma Lattes, criando relatórios e gráficos no formato HTML (Hypertext MarkupLanguage, ou em português, Linguagem de Marcação de Hipertexto), contendo os indicadores de produção intelectual de todos os pesquisadores listados previamente (ALVES; SANTOS; SCHIMIT, 2016).

\section{METODOLOGIA}

O presente estudo visa apresentar os indicadores de produtividade acadêmica de um grupo de docentes de cursos de graduação de uma IES, mensurando, quantitativamente e qualitativamente, sua produção intelectual, demonstrando a responsabilidade social e a transparência/popularização das pesquisas deste grupo de professores.

Diante desse contexto, considera-se esta pesquisa como qualitativa exploratória, por abordar um tema pouco explorado, que é a análise da produtividade acadêmica dos docentes de cursos de graduação de IES, contribuindo para o entendimento do fenômeno, e auxiliando na construção de pesquisas futuras sobre o tema (SAMPIERI et al., 2006).

Outra abordagem do presente estudo é a descrição do funcionamento operacional do Scriptemec, uma ferramenta computacional que tem como objetivo a extração e organização dos dados referentes à produtividade acadêmica de docentes de cursos de graduação, destacando-se os procedimentos prévios para a execução da ferramenta, o processo de extração, assim como a forma de apresentação dos dados obtidos na Plataforma Lattes. Portanto, esta pesquisa possui uma abordagem qualitativa descritiva, em que o fenômeno é interpretado e detalhado, sem juízo de valor do pesquisador, compreendendo todo o processo de construção do conhecimento (PEROVANO, 2014).

Para apresentar a eficácia do uso da ferramenta Scriptemec para a avaliação contínua e automatizada da produtividade docente, definiu-se alguns critérios para uma escolha alinhada com os objetivos propostos da pesquisa. Os critérios para a definição da IES estudada foram: 
1 - Possuir cursos de graduação com possibilidade de renovação de reconhecimento e/ou passíveis de autorização, conforme diretrizes definidas pelo INEP/MEC;

2 - Possuir valores institucionais relacionados à responsabilidade social e auxílio a comunidade local;

3 - Ter um corpo docente formado por Mestres e Doutores, auxiliando na análise do pilar Corpo Docente e Tutorial, conforme o "instrumento" estabelecido pelo INEP;

4 - Ter o curso de graduação em Medicina dentro do portifólio acadêmico, tendo em vista que este curso possui critérios específicos de análise pelo "instrumento";

5 - Disponibilizar as informações necessárias sobre a produtividade acadêmica do corpo docente dos cursos de graduação. Assim, definiu-se como objeto deste estudo a IES Alfa (a IES não autorizou a utilização de seu nome real, sendo a partir deste momento do trabalho designada por Alfa), que se enquadra em todos os critérios supracitados.

A Alfa se trata de uma IES privada, sendo composta por 9 campi distribuídos nos estados de São Paulo (6), Paraná (1), Rio de Janeiro (1) e Minas Gerais (1). Embora a instituição ofereça desde Cursos Abertos até Pós-Graduação Stricto sensu (Mestrado e Doutorado Acadêmico), o foco deste trabalho será os cursos de graduação, que são oferecidos somente nos campi de São Paulo. A Alfa, que é uma IES focada em Ciências da Saúde, possui somente dois cursos de graduação, sendo estes a Enfermagem, que se enquadra na possibilidade de renovação de reconhecimento, e a Medicina, que encaixa-se no âmbito de autorização do curso, cumprindo os critérios 1 e 4 para definição desta IES como objeto do presente estudo. Como a Alfa faz parte de um sociedade beneficente, e que apresenta entre seus valores institucionais a Responsabilidade Social e o Apoio à Comunidades Carentes, esta IES atende ao critério 2 de seleção. Para cumprir o critério 3, a Alfa possui somente Mestres e Doutores como docentes de seus cursos de graduação. Por fim, a IES permitiu o acesso aos dados acadêmicos e profissionais dos 45 professores de seus cursos de graduação, cumprindo o critério 5, e justificando sua escolha.

A fim de obter os dados atualizados da produtividade acadêmica dos professores por meio da execução do Scriptemec, foram coletados os seguintes dados dos docentes da Alfa: Nome, Tempo da Contratação Profissional, Curso ao qual está vinculado atualmente, Matrícula Institucional e ID Lattes, para iniciar a análise na Fase Diagnóstica. Além disso, utilizou-se a Plataforma Lattes para se obter os dados de produtividade acadêmica deste 
grupo, como por exemplo, a Produção Bibliográfica, Produção Técnica, Projetos de Pesquisa, Orientações e Supervisões em Andamento, entre outros indicadores.

Neste parágrafo apresenta-se o procedimento para a coleta dos dados do grupo de docentes da presente pesquisa. Inicialmente, construiu-se a lista de entrada no formato ".csv" para execução do Scriptemec, possibilitando a extração, organização e apresentação dos dados de produtividade acadêmica do corpo docente, para sua posterior análise. Esta lista de entrada em “.csv” possuía a seguinte ordem de informações dos docentes: ID Lattes, Matrícula, Nome Completo, Programa/Curso Vinculado (Enfermagem ou Medicina), Período de Início de Contratação, Período Fim de Contratação (caso existisse). Concomitantemente, foi criado um arquivo de configurações com a extensão ".txt” para criar uma hierarquia das informações que seriam extraídas pelo Scriptemec. Na sequência, ocorreu o agrupamento hierárquico das informações, gerando os scripts que foram executados pelo Scriptlattes. Posteriormente, foram executados os Scriptlattes, por meio do Scriptemec, extraindo-se todos os currículos dos docentes previamente listados. Na próxima etapa do processo, o Scriptlattes organizou todos os dados acadêmicos dos docentes no período solicitado (1979 até Abril/2017), como produção bibliográfica, produção técnica, orientações em andamento, etc., tratando as redundâncias ligadas à estas produções por meio da exclusão de sobreposições de títulos. Estes scripts geraram arquivos com a extensão “.list", contendo os IDs Lattes e nome dos professores, e com a extensão “.config”, possuindo os dados de produtividade acadêmica registrados por cada docente na Plataforma Lattes. Por último, após nova execução do Scriptlattes com os arquivos supracitados, foram obtidos os relatórios com os indicadores de produtividade acadêmica do grupo pesquisado, sendo apresentados em formato $H T M L$, e gerando o Relatório Completo Agrupado, para sua posterior análise. Na Figura 2 são apresentadas, resumidamente, as etapas de funcionamento do Scriptemec, para extração dos indicadores de produtividade acadêmica dos pesquisadores de cursos de graduação da Alfa.

Após a extração e organização dos dados acadêmicos dos docentes dos cursos de graduação da Alfa, realizadas pelo Scriptemec, todos os indicadores de produção bibliográfica, produção técnica, produção artística, orientações, projetos de pesquisa, prêmios e títulos, participação em eventos e organização de eventos, referente aos docentes dos cursos de graduação da Alfa, foram analisados de forma quantitativa. Concomitantemente, estes mesmos dados foram analisados de forma qualitativa, com base na avaliação do nível de 
responsabilidade social das pesquisas conduzidas pelos professores da IES, e da transparência/popularização destas pesquisas.

Figura 2 Apresentação resumida das etapas de funcionamento do Scriptemec

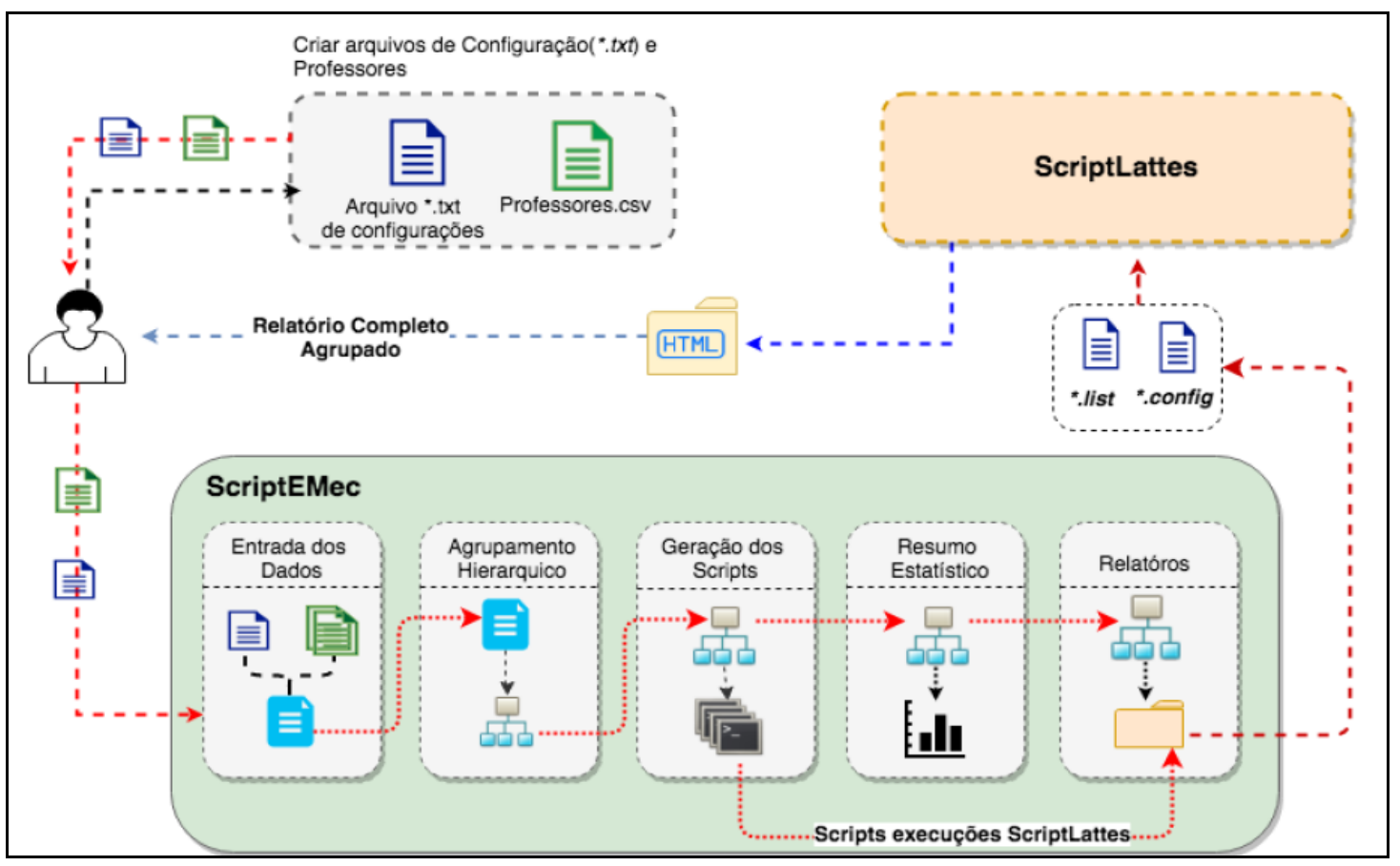

Fonte: Santos (2017).

\section{ANÁLISE DOS RESULTADOS}

A Figura 3 se refere à tela inicial, disponibilizada pelo Scriptemec após a extração dos dados profissionais e acadêmicos dos docentes cadastrados na Plataforma Lattes. Nessa tela, é possível visualizar os links de acesso às páginas com os indicadores de produção acadêmica dos professores avaliados, bem como as produções por curso e por ano.

Figura 3 Página inicial com os links de acesso aos resultados gerados pelo Scriptemec

\section{CURSOS ALFA}

\footnotetext{
- Egressos

- Enfermagem

- Medicina
}

Por periodo: 1989, 1990, 1991, 1992, 1993, 1994, 1995, 1996, 1997, 1998, 1999, 2000, 2001, 2002, 2003, 2004, 2005, 2006, 2007, 2008, 2009, 2010, 2011, 2012, 2013, 2014, 2015, 2016, 2017, Periodo Triênio 2014-2016

ScriptEmecø. ScriptLattese

Fonte: http://ec2-35-164-224-104.us-west-2.compute.amazonaws.com/scriptdataset-result .

Ao acessar o link CURSOS ALFA, indicado pela seta vermelha na Figura 3, ocorre o direcionamento para a tela apresentada na Figura 4, em que é possível identificar todos os 
links de acesso a cada um dos indicadores relacionados à produção bibliográfica, produção técnica, produção artística, orientações conduzidas pelos professores, projetos de pesquisa, prêmios e títulos, e participação e organização de eventos.

Figura 4 Tela com links de acesso aos diferentes tipos de produção dos professores avaliados

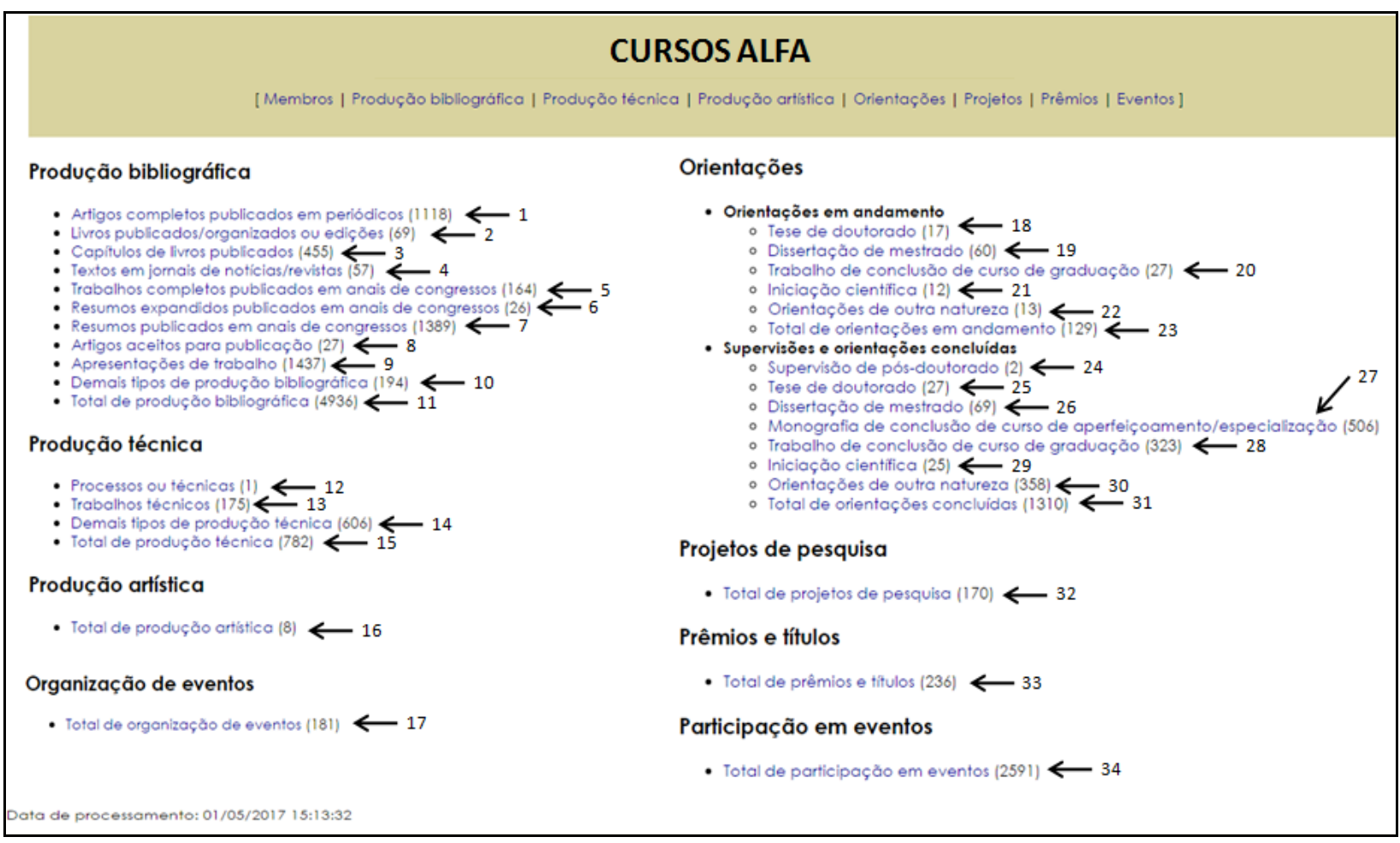

Fonte: http://ec2-35-164-224-104.us-west-2.compute.amazonaws.com/scriptdataset-result.

Como no presente artigo pretende-se contribuir com a apresentação do Scriptemec como ferramenta de gestão estratégica do desempenho relacionado a produção intelectual dos professores de cursos de graduação das IES, optou-se por destacar os indicadores que permitem o acompanhamento da produtividade acadêmica dos docentes ao longo de um período desejado, avaliar os níveis de transparência/popularização das pesquisas destes professores, e analisar a responsabilidade social dos estudos promovidos pelos pesquisadores ligados aos cursos de graduação da IES objeto do presente estudo.

Assim, na Figura 5, apresenta-se os resultados presentes no link "Artigos completos publicados em periódicos" (seta 1, na Figura 4). Nota-se que no período de 1984 à Abril / 2017, o número de artigos publicados totalizou 1.118 produções, distribuídas ano à ano, o que permite uma análise histórica da produtividade acadêmica dos docentes da Alfa. Ainda reforça-se a presença da coluna *itens sem ano (1), que representa o registro incorreto de publicações na Plataforma Lattes pelos próprios docentes, o que pode gerar à Alfa uma 
insconsistência na prestação de contas ao INEP, e a consequente redução da nota do curso avaliado por este órgão governamental.

Figura 5 Tela com a representação gráfica dos "Artigos completos publicados em periódicos"

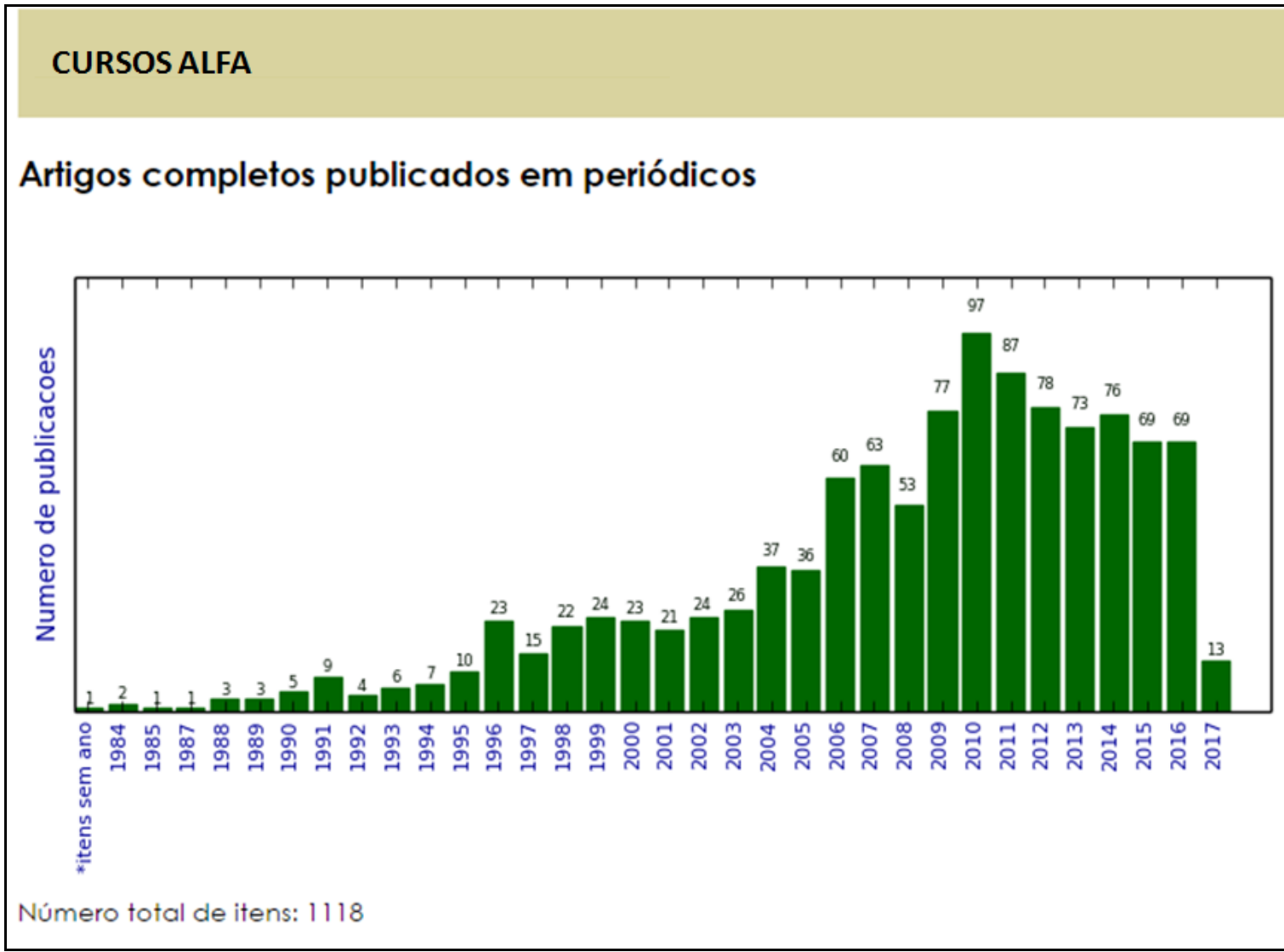

Fonte: http://ec2-35-164-224-104.us-west-2.compute.amazonaws.com/scriptdataset-result.

Já na Figura 6, demonstra-se o indicador "Textos em jornais de notícias/revistas" (seta 4, na Figura 4), em que é possível verificar o índice de popularização/transparência das pesquisas publicadas pelos docentes dos cursos de graduação da Alfa. No período de 1995 até 2013, a IES teve 57 publicações, sendo que 21\% do total (12 publicações) não foram registradas corretamente na Plataforma Lattes, sendo representadas como *itens sem ano. Como já dito anteriormente, este erro pode prejudicar a avaliação institucional da Alfa, além de não permitir que a IES mensure corretamente este indicador qualitativo muito importante na análise da produtividade acadêmica de uma IES (FERRAZ et al., 2017). 
Figura 6 Tela com a representação gráfica dos indicadores de "Textos em jornais de notícias/revistas"

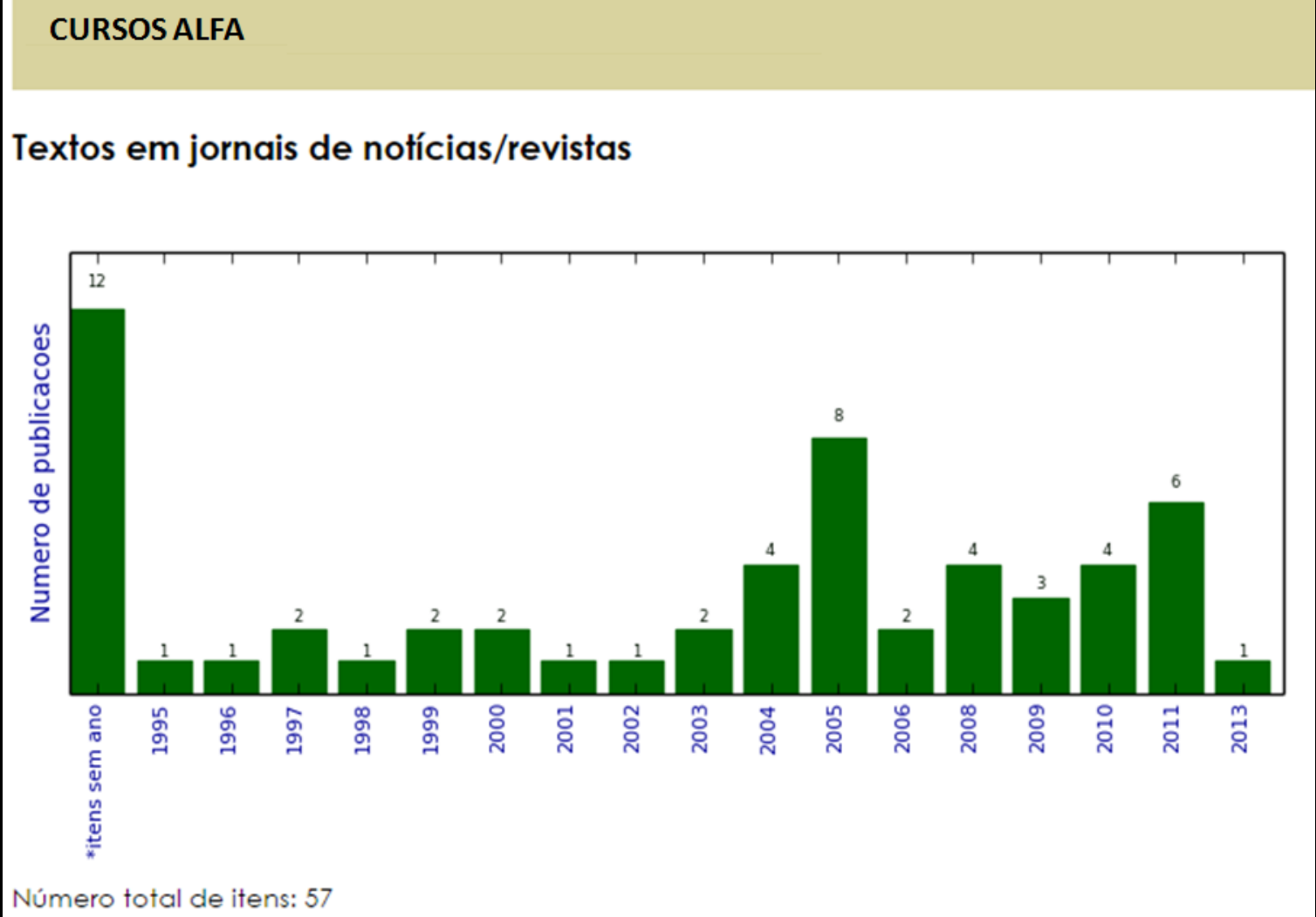

Fonte: http://ec2-35-164-224-104.us-west-2.compute.amazonaws.com/scriptdataset-result.

Por fim, na Figura 7, identifica-se o indicador “Total de produção técnica” (seta 15, na Figura 4), que é representado pelo gráfico de barras com a distribuição das 782 produções publicadas pelos professores da Alfa, no período de 1984 até 2016. Por meio deste indicador, mensura-se o nível de responsabilidade social presente nas pesquisas da IES (FERRAZ et al., 2016). Os mesmos autores destacam que a produção científica em IES leva o conhecimento acadêmico até a comunidade, contribuindo para a produção do capital humano e intelectual, além de incrementar o desenvolvimento tecnológico do país. Nesse sentido, entende-se a importânica na análise deste indicador por parte das IES, além de ser essencial na prestação de contas ao INEP, que ocorre periodicamente conforme calendário definido pelo MEC. 
Figura 7 Tela com a representação gráfica dos indicadores de "Total de produção técnica"

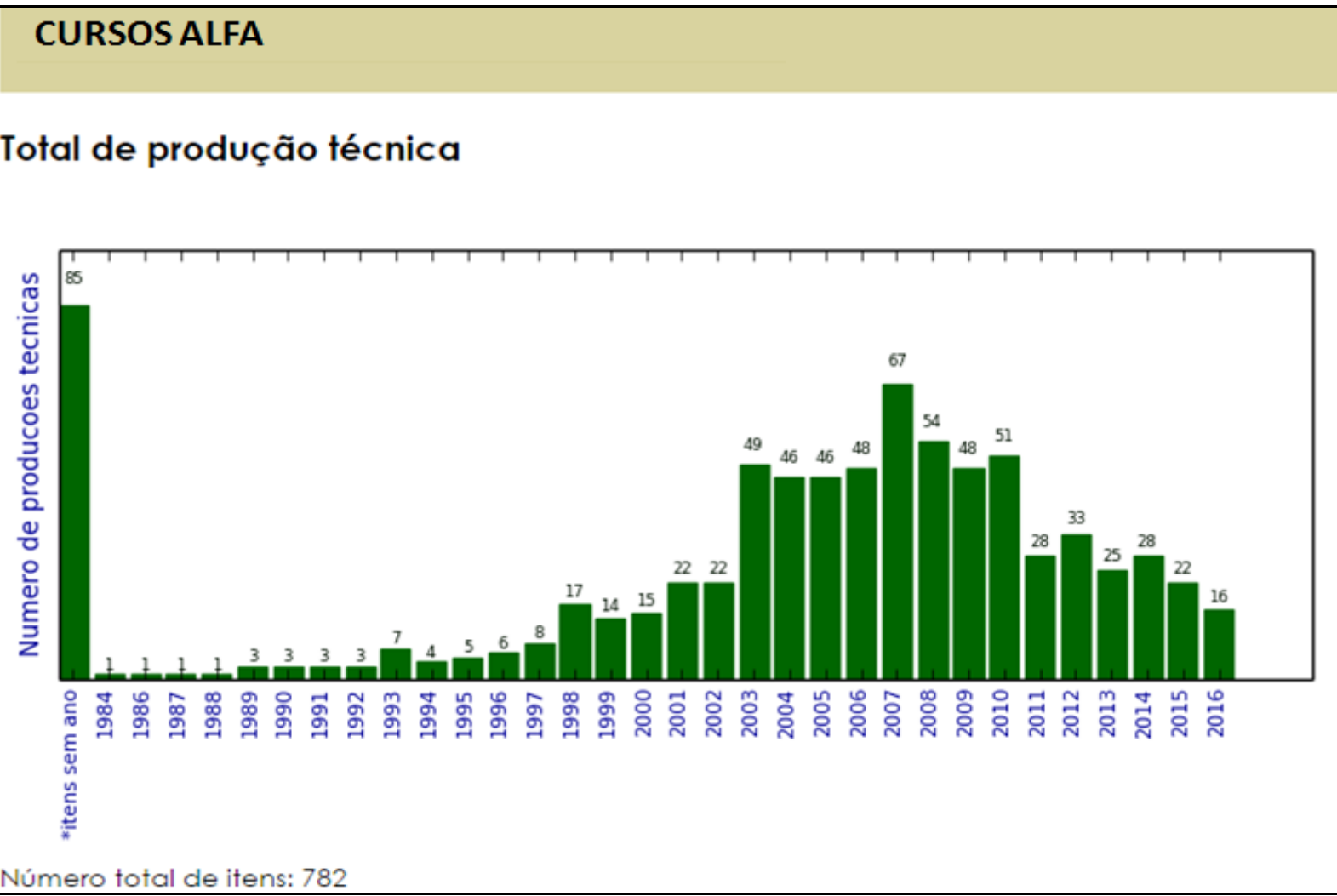

Fonte: http://ec2-35-164-224-104.us-west-2.compute.amazonaws.com/scriptdataset-result.

\section{CONSIDERAÇÕES FINAIS}

Com o uso da ferramenta computacional Scriptemec, entende-se que os gestores acadêmicos dos cursos de graduação da IES avaliada podem acompanhar, continuamente, a produtividade acadêmica do corpo docente. Dessa forma, foi possível avaliar e gerenciar de forma estratégica os diversos indicadores de produtividade, tão importantes no momento de prestação de contas ao INEP. Acredita-se também que é possível, com base nas informações fornecidas pela ferramenta, executar em tempo hábil planos de ação visando alavancar a produtividade científica dos professores, quando necessário. Dessa forma, sanções promovidas pelo INEP poderão ser evitadas, como por exemplo, a redução da nota de avaliação, ou mesmo o descredenciamento dos cursos de graduação, o que traria prejuízos institucionais e financeiros às IES.

Ademais, a automatização do processo de extração, organização e apresentação dos dados de produtividade em pesquisa do corpo docente, permitiram uma melhor gestão de diversos recursos da IES. Dentre os pontos de melhoria, podem ser citados a otimização do tempo dos funcionários técnico-administrativos, que poderão exercer outras funções ao invés 
de gastar tempo realizando a coleta manual de dados da Plataforma Lattes para montagem de planilhas que são conferidas, a posteriori, pelos gestores de cada curso, e também reduzindo ainda a possibilidade de erros durante a organização manual das informações coletadas.

Outro ponto importante se refere à gestão acadêmica, focada no acompanhamento da produção científica dos pesquisadores, visto que a automatização dos processos de acompanhamento da produtividade docente permite o controle desta importante capacidade interna, o que pode influenciar positivamente na vantagem competitiva. Entende-se também, que o processo de automatização da gestão da produtividade em pesquisa com a utilização da ferramenta computacional Scriptemec, permitiu o desenvolvimento de uma cultura inovativa no segmento de ensino superior, com uma utilização mais consciente dos recursos das IES.

Após a presente pesquisa, recomenda-se a execução de pesquisas futuras que identifiquem ações para uma melhoria no preenchimento da Plataforma Lattes, o que pode auxiliar na resolução das inconsistências nos indicadores de produtividade acadêmica. Com isso, a efetividade no uso do Scriptemec poderá ser ainda mais evidente, por assim conseguir apresentar dados atualizados e corretos dos pesquisadores. Outra sugestão, é o acompanhamento anual e trienal, período avaliativo do INEP, dos indicadores de produtividade acadêmico dos docentes de curso de graduação com o auxílio do Scriptemec, a fim de reforçar o uso da ferramenta no processo da gestão estratégica das IES brasileiras.

Por fim, esta pesquisa têm como contribuição teórica a possibilidade do estudo do desempenho acadêmico em pesquisa de docentes de cursos de graduação com o apoio de uma ferramenta estratégica de livre acesso e que permite uma otimização na análise de indicadores de produtividade intelectual. Como contribuição acadêmica, trata-se de uma nova forma de analisar um assunto ainda pouco explorado nos cursos de graduação (MEYER; PASCUCCI; ANDRIGUETTO, 2017), que é a avalição estratégica dos indicadores de produtividade acadêmica dos pesquisadores, impactando o valor institucional de ensino e pesquisa das IES do Brasil.

\section{REFERÊNCIAS}

ALVES, Alexandre Donizeti; YANASSE, Horacio Hideki; SOMA, Nei Yoshihiro. LattesMiner: uma linguagem de domınio especıfico para extraçao automática de informações da Plataforma Lattes. In: 2012, São José dos Campos. Anais... . In: XII WORKSHOP DE COMPUTAÇÃO APLICADA. São José dos Campos 
ALVES, Wonder; SANTOS, Saulo D.; SCHIMIT, Pedro H. T. Hierarchical Clustering Based on Reports Generated by Scriptlattes. In: NÄÄS, Irenilza et al. (Eds.). Advances in Production Management Systems. Initiatives for a Sustainable World. Cham: Springer International Publishing, 2016. v. 488p. 28-35.

BAETA, Odemir V.; BRITO, Mozar J.; SOUZA, Rosalia B. Strategy as Discursive Practice in a Brazilian Public University: A Look under the Perspective of Critical Discourse Analysis. Public Administration Research, [s. 1.], v. 3, n. 2, p. 17-27, 2014.

BARNEY, Jay. Firm Resources and Sustained Competitive Advantage. Journal of Management, [s. 1.], v. 17, n. 1, p. 99-120, 1991.

BARREYRO, Gladys Beatriz. De exames, rankings e mídia. Avaliação: Revista da Avaliação da Educação Superior (Campinas), [s. 1.], v. 13, n. 3, p. 863-868, 2008.

BAVARESCO, Juliano. A Ferramenta Computacional Scriptemec como Estratégia de Gerenciamento da Produtividade Acadêmica de uma Instituição Privada de Ensino Superior. 2017. Dissertação - Universidade Nove de Julho, São Paulo, 2017.

BERNARDES, Joelma Dos Santos; ROTHEN, José Carlos. O campo da Avaliação da Educação Superior: foco na Comissão Própria de Avaliação. Revista Meta: Avaliação, [s. 1.], v. 8, n. 23, p. 248, 2016.

BRITO, Márcia Regina F. De. O SINAES e o ENADE: da concepção à implantação. Avaliação: Revista da Avaliação da Educação Superior (Campinas), [s. 1.], v. 13, n. 3, p. 841-850, 2008.

CANAN, Silvia Regina; ELOY, Vanessa Tais. Políticas de avaliação em larga escala: o ENADE interfere na gestão dos cursos? Praxis Educativa, [s. 1.], v. 11, n. 3, p. 621-640, 2016.

CAPES. Coordenação de Aperfeiçoamento de Pessoal de Nível Superior. 2016. Disponível em: <http://www.capes.gov.br/>. Acesso em: 13 set. 2016.

CARDOSO, Sónia; ROSA, Maria J.; STENSAKER, Bjørn. Why is quality in higher education not achieved? The view of academics. Assessment \& Evaluation in Higher Education, [s. 1.], v. 41, n. 6, p. 950-965, 2016.

CASTRO, Sabrina Olimpio Caldas De et al. A Influência do ENADE no âmbito das Instituições de Ensino Superior. Educação, Ciência e Cultura, [s. 1.], v. 21, n. 1, p. 23-41, 2016.

CESSO, Marcus Vinicius; FERRAZ, Renato Ribeiro Nogueira. Utilização do ENADE (Exame Nacional de Desempenho de Estudantes) como ferramenta de avaliação do desempenho de alunos de uma Instituição de Ensino Superior paulistana. Revista de Gestão e Secretariado, [s. 1.], v. 8, n. 3, p. 93-112, 2017. 
CNPQ. Plataforma Lattes. 2016. Disponível em: $<$ http://lattes.cnpq.br/>. Acesso em: 30 nov. 2016.

DIMAGGIO, Paul Joseph; POWELL, Walter W. A gaiola de ferro revisitada: isomorfismo institucional e racionalidade coletiva nos campos organizacionais. RAE - Revista de Administração de Empresas, [s. 1.], v. 45, n. 2, p. 74-89, 2005.

FERRAZ, Renato Ribeiro Nogueira et al. Uso da ferramenta computacional Scriptsucupira para gestão estratégica da produtividade em pesquisa de um programa de Pós-Graduação Stricto sensu em biofotônica. Revista de Administração da Unimep, [s. 1.], v. 14, n. 3, p. 232-252, 2016.

FERRAZ, Renato Ribeiro Nogueira et al. Planejamento anual e quadrienal de prestação de contas à Capes por meio da ferramenta computacional Scriptsucupira. Revista Brasileira de Pós-Graduação, [s. 1.], v. 14, n. 0, p. 1-25, 2017.

FERREIRA, Vinicius Marques et al. A Autoavaliação institucional no ensino superior como técnica de gestão estratégica. Revista Edu. Tec., [s. 1.], v. 7, n. 1, 2016.

GOMES, Válter et al. A Estratégia como prática nas Instituições de Ensino Superior. Revista Evidência, [s. 1.], v. 11, n. 11, p. 115-126, 2015.

HOFFMANN, Celina et al. O desempenho das universidades brasileiras na perspectiva do Índice Geral de Cursos (IGC). Educação e Pesquisa, [s. 1.], v. 40, n. 3, p. 651-665, 2014.

LACERDA, Leo Lynce Valle De; FERRI, Cássia; DUARTE, Blaise Keniel da Cruz. SINAES: avaliação, accountability e desempenho. Revista da Avaliação da Educação Superior, [s. 1.], v. 21, n. 3, p. 975-992, 2016.

MACCARI, Emerson Antonio et al. Proposta de um modelo de gestão de programas de pósgraduação na área de Administração a partir dos sistemas de avaliação do Brasil (CAPES) e dos Estados Unidos (AACSB). Revista de Administração - RAUSP, [s. 1.], v. 49, n. 2, p. 369-383, 2014.

MARCHELLI, Paulo Sérgio. O sistema de avaliação externa dos padrões de qualidade da educação superior no Brasil: considerações sobre os indicadores. Estudos em avaliação educacional, [s. 1.], v. 18, n. 37, p. 189-216, 2007.

MARTIN, Michaela. External quality assurance in higher education: how can it address corruption and other malpractices? Quality in Higher Education, [s. 1.], v. 22, p. 49-63, 2016.

MARTINS, Cibele Barsalini et al. Influência das estratégias e recursos para o desenvolvimento dos programas de pós-graduação da área de Administração, Ciências Contábeis e Turismo no período de 2001 a 2009. Revista Gestão Universitária na América Latina - GUAL, [s. 1.], v. 6, n. 3, p. 146-168, 2013. 
MEC. Ministério da Educação. Home. 2017. Disponível em: <http://portal.mec.gov.br/>. Acesso em: 16 mar. 2017.

MENA-CHALCO, Jesus Pascual; JUNIOR, Roberto Marcondes Cesar. ScriptLattes: an opensource knowledge extraction system from the Lattes platform. Journal of the Brazilian Computer Society, [s. 1.], v. 15, n. 4, p. 31-39, 2009.

MEYER, Victor; PASCUCCI, Lucilaine; ANDRIGUETTO, Haroldo. Estrategias académicas: conflictos en la gestión de un sistema complejo. Revista ESPACIOS, [s. 1.], v. 38, n. 04, 2017.

PEROVANO, Dalton Gean. Manual de Metodologia Científica. 1. ed. [s.1.] : Juruá Editora, 2014.

POLIDORI, Marlis Morosini et al. Políticas de Avaliação da Educação Superior Brasileira. Educação \& Realidade, [s. 1.], v. 36, n. 1, p. 253-278, 2011.

POLIDORI, Marlis Morosini; CARVALHO, Nathan Ono De. Acreditação de Instituições de Educação Superior: uma necessidade ou uma normatização. Avaliação: Revista da Avaliação da Educação Superior, [s. 1.], v. 21, n. 3, 2016.

RODRIGUES, Simone et al. Mapeamento dos grupos de pesquisa do CNPq na área de Engenharias e Computação. Revista Uniabeu, [s. 1.], v. 10, n. 24, p. 246-255, 2017.

SAMPIERI, Roberto Hernández et al. Metodologia de pesquisa. 5. ed. [s.1.] : McGraw Hill, 2006.

SANTO, Eniel do Espirito; TRAVASSOS, Xisto Lucas; CARIBÉ, Sabrina Oliveira. Análise do nível de implantação do processo de autoavaliação nas faculdades privadas de Salvador, Bahia. Revista da Avaliação da Educação Superior (Campinas), [s. 1.], v. 21, n. 1, p. 153$172,2016$.

SILVA, Marcus Vinicius Cesso Da; FERRAZ, Renato Ribeiro Nogueira. Aplicação do ENADE como ferramenta para avaliação do desempenho discente em um curso de Pedagogia. Revista Eletrônica de Educação, [s. 1.], v. 12, n. 1, p. 74-89, 2018.

SILVA, Marcus Vinicius Cesso. Avaliação contínua e automatizada da produtividade acadêmica dos cursos de graduação de uma instituição de ensino superior. 2017. Dissertação - Universidade Nove de Julho, São Paulo, 2017.

SILVA, Marcus Vinicius Cesso; FERRAZ, Renato Ribeiro Nogueira. Use of computational tools for strategic management of research productivity in graduation Stricto sensu program: a systematic literature review. In: CONTECSI 2017 2017, São Paulo. Anais... . In: XIV CONTECSI. São Paulo: FEA, 2017.

SOARES, Thiago Coelho; ROMAN, Darlan José; ERDMANN, Rolf Hermann. Fatores de Competitividade na Educação Superior. Gestão Contemporânea, [s. 1.], n. 18, p. 56-73, 2016.

SOBRINHO, José Dias. Avaliação e transformações da educação superior brasileira (19952009): do Provão ao SINAES. Avaliação: Revista da Avaliação da Educação Superior, [s. 1.], v. 15, n. 1, 2010. 